\title{
Brachydactyly-mesomelia-intellectual disability-heart defects syndrome
}

INSERM

\section{Source}

INSERM. (1999). Orphanet: an online rare disease and orphan drug data base.

Brachydactyly-mesomelia-intellectual disability-heart defects syndrome. ORPHA:1277

Brachydactyly-mesomelia-intellectual disability-heart defects syndrome is a rare, genetic, multiple congenital anomalies/dysmorphic syndrome characterized by developmental delay, intellectual disability, thin habitus with narrow shoulders, mesomelic shortness of the arms, craniofacial dysmorphism (e.g. long lower face, maxillary hypoplasia, beak nose, short columella, prognathia, high arched palate, obtuse mandibular angle), brachydactyly (mostly involving middle phalanges) and cardiovascular anomalies (i.e. aortic root dilatation, mitral valve prolapse). 